\title{
INDICAÇÃO DE ESPÉCIES DE PORTE ARBÓREO PARA A ARBORIZAÇÃO URBANA NO SEMI-ÁRIDO PARAIBANO
}

\author{
Roberta Maria de Albuquerque Lacerda'; José Augusto de Lira Filho²; Rivaldo Vital dos \\ Santos ${ }^{3}$
}

(recebido em 23.09.2010 e aceito para publicação em 15.03.2011)

\section{RESUMO}

O maior desafio da arborização urbana está no conflito que se estabelece entre as árvores e os demais elementos que compõem o ambiente. Em ambientes que apresentam um clima hostil, como ocorre em cidades do semi-árido brasileiro, esse desafio é bem maior. A solução depende, no ato do planejamento, da escolha correta das árvores. Para isto, procederam-se pesquisas no intuito de se identificar e analisar as potencialidades de espécies de porte arbóreo, nativas ou exóticas adaptadas, que possam ser indicadas adequadamente para a arborização urbana. Os estudos foram realizados no sertão paraibano, tendo-se identificado 29 espécies, nativas e exóticas, cujas potencialidades foram analisadas, resultando em 21 indicações para arborização de vias públicas, 29 para áreas verdes e 07 para mata ciliar. Do total de árvores pesquisadas e selecionadas, 7 são de pequeno porte, 14 de porte médio e 8 de grande porte.

Palavras-Chave: árvores urbanas, silvicultura urbana, Sertão Nordestino.

\footnotetext{
1 Mestre em Ciências Florestais formada pela Universidade Federal de Campina Grande, Patos-Paraíba, robertaufcg@yahoo.com.br

2 Doutor em Paisagismo, Professor Adjunto da Unidade Acadêmica de Engenharia Florestal/ Universidade Federal de Campina Grande, Patos-Paraíba, augustoufcg@yahoo.com.br.

3 Doutor em Solos e Nutrição de Plantas, Professor Adjunto da Unidade Acadêmica de Engenharia Florestal/ Universidade Federal de Campina Grande, Patos-Paraíba, rvital@cstr.ufcg.edu.br
} 


\section{INDICATION OF TREE SPECIES TO THE URBAN FOREST IN SEMI-ARID CLIMATE OF PARAÍBA}

\section{ABSTRACT}

The biggest challenge of urban forest is in the conflict that happens between trees and other elements that make up the urban environment. Challenge is bigger in environments with a hostile climate such as the cities of Brazilian semi-arid region. In act of planning solution depends the correct choice of trees. Thus, researches were done aiming to identify and analyze potentialities of tree species, native or adapted exotic that could be correctly indicated for urban forest. Studies were conducted in semi-arid of State of Paraíba, Brazil, where 29 native and exotic species were identified, whose potentialities were analyzed, resulting in 21 indications for urban forest of public streets, 29 for green areas and 7 for riparian forest. Of all researched and selected trees, 7 are small size, 14 are medium size and 8 are large size.

Keywords: urban trees, urban forestry, Northeastern Wilderness.

\section{INTRODUÇÃO}

O semi-árido brasileiro concentra-se em quase sua totalidade na região Nordeste, compreendendo o interior de todos os Estados nordestinos e se estende até o norte de Minas Gerais. Isto equivale a uma ocupação territorial de $1.150 .662 \mathrm{~km}^{2}$ correspondente a $13,52 \%$ do território brasileiro e $74,30 \%$ da área do Nordeste.

A cobertura vegetal dessa região é constituída por formações típicas denominadas de Caatinga ou Savana Estépica, caracterizada pela formação de floresta seca composta de vegetação xerófila de porte arbóreo, arbustivo e herbáceo, com ampla variação de fisionomia em estratos arbóreo, arbustivo e herbáceo, com predominância de espécies das famílias Caesalpinaceae, Mimosaceae, Euphorbiaceae, Fabaceae e Cactacea (SUDEMA, 2004). Estas espécies vegetais são exploradas, aleatoriamente, para os mais diversos usos, levando-as quase a extinção pelo desconhecimento do manejo e usos adequados. Tal 
desconhecimento constitui-se em impedimento para a exploração das reais potencialidades da vegetação nativa.

Nos ecossistemas urbanos, inseridos no semi-árido, algumas espécies nativas são utilizadas na arborização de vias públicas, porém pouco se conhece sobre as suas potencialidades que permitam uma escolha adequada, obedecendo a todos os critérios mencionados por Gonçalves e Paiva (2004) relacionados ao porte, frutescência, florescência, tipos de raízes, e ritmo de crescimento. Sabe-se que as cidades do semi-árido apresentam um ambiente hostil para implantação da vegetação arbórea, sobretudo pelas limitações climáticas. Submetidas a um clima quente e seco, com até nove meses de estiagem, essas cidades necessitam da presença de uma massa arbórea que lhes proporcione melhorias no padrão ambiental, garantindo-lhes uma melhoria na qualidade de vida das populações nelas inseridas.

As árvores urbanas proporcionam diversos benefícios ecológicos, psicológicos, econômicos e sociais para a população e, especificamente para as cidades do semi-árido, elas desempenham função preponderante para a estabilização e melhoria microclimática, reduzindo a radiação solar direta e aumentando o conforto térmico (GONÇALVES e PAIVA, 2004).

Neste contexto, considerando-se a importância das árvores urbanas para melhoria da qualidade de vida e elevação do padrão sócio-ambiental pelas diversas funções que exercem no ecossistema urbano, esta pesquisa se propôs a identificar e analisar as potencialidades de espécies de porte arbóreo, nativas ou exóticas adaptadas à região, que possam ser indicadas adequadamente para a arborização urbana.

Esta pesquisa foi direcionada para espécies vegetais de porte arbóreo que, segundo Gonçalves (1999), se diferenciam das demais plantas por serem de maior porte, lenhosas e de tronco único. Nesta classificação, englobam-se tanto as árvores ditas folhosas, que se caracterizam pelo crescimento simpodial, quanto às coníferas que se caracterizam pelo crescimento misto (simpodial e monopodial), bem como as palmeiras, de crescimento monopodial.

Para o pleno sucesso do Plano Diretor de Arborização é de fundamental importância a escolha da espécie adequada para implantação no ecossistema urbano. Segundo Gonçalves e Paiva (2004), uma escolha bem feita significa o sucesso do plano, além da diminuição dos onerosos gastos com tratos culturais e manutenção de árvores que foram implantadas em locais inadequados e sem um mínimo de planejamento anterior.

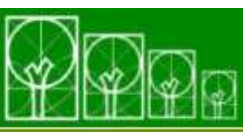

S $\cdot \mathbf{B} \cdot \mathbf{A} \cdot \mathbf{U}$ Soc. Bras. de Arborização Urbana 
Essa falta de planejamento da arborização com bases técnico-científicas pode ocasionar vários transtornos e problemas nas cidades. Para isto, faz-se necessário à utilização de critérios para adequar cada árvore a um determinado ambiente urbano (GONÇALVES e PAIVA, 2004; MILANO e DALCIN, 2000). Tais critérios se referem tanto aos aspectos ambientais quanto aos relacionados à vegetação a ser implantada.

No contexto estético, conforme Lira Filho (2002), as árvores urbanas podem ser analisadas sob os seguintes parâmetros: forma, textura e cor. Segundo este autor, na paisagem urbana a vegetação com as suas mais variadas formas adquire uma significação toda especial para os espectadores e usuários. Quanto à textura, o paisagista pode tirar partido de uma série de texturas encontradas na conformação da copa e do tronco das árvores.

\section{Abrangência territorial da pesquisa}

No que se refere à abrangência territorial urbana, a pesquisa foi realizada em um raio de $6.037,7 \mathrm{~km}^{2}$ correspondente ao Território do Médio Sertão Paraibano, situado nas mesorregiões geográfica do Sertão da Paraíba e Borborema, composto pelas seguintes microrregiões: Patos, Seridó Ocidental, Sousa, Piancó, e Serra do Teixeira (CODETER MÉDIO SERTÃO, 2005). 


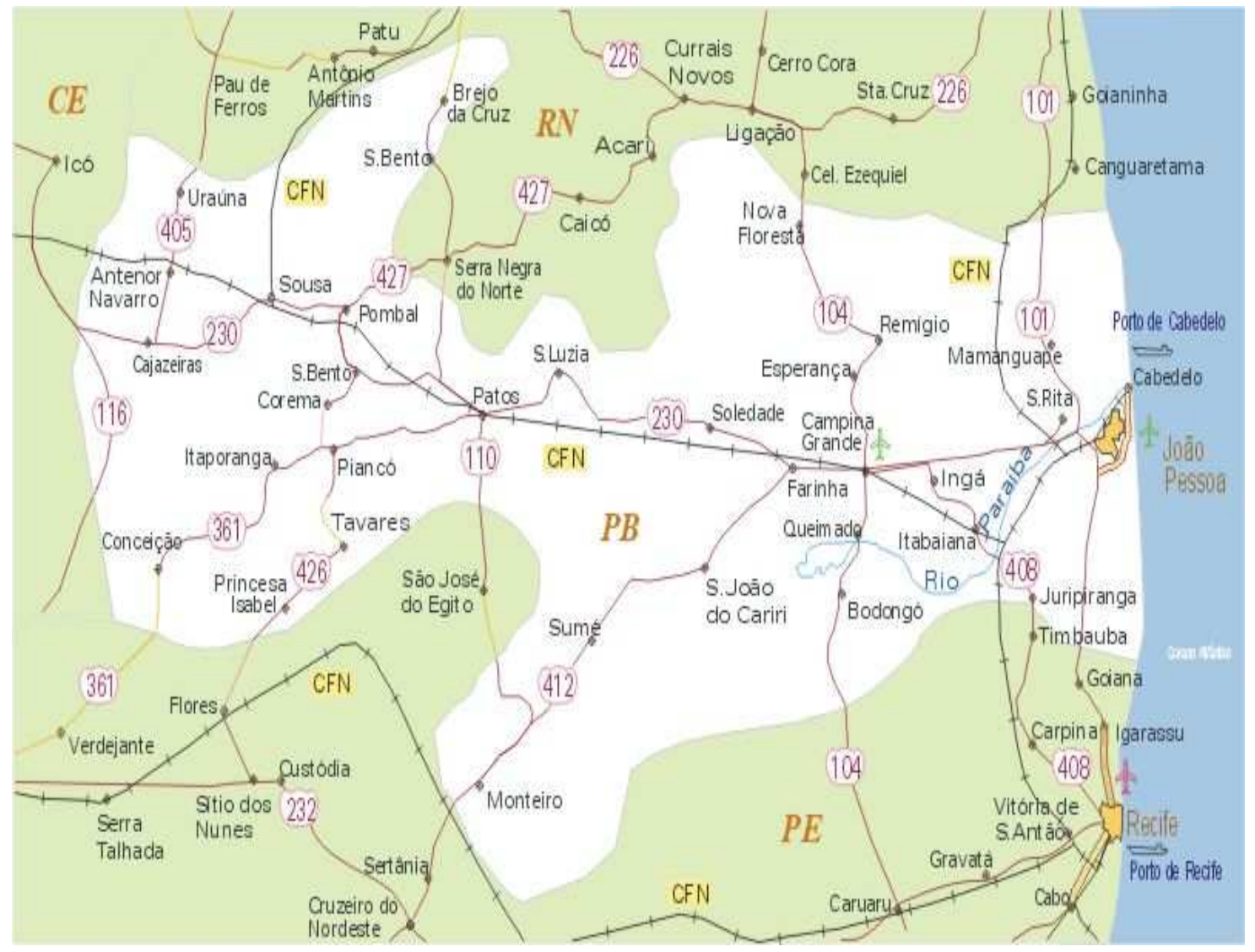

FIGURA 1 - Mapa do Estado da Paraíba com a localização das áreas de estudo.

FIGURE 1 - Map of State of Paraíba with studied areas localization.

A pesquisa foi realizada no período de 2005 a 2007, incluindo áreas urbanas e fronteiriças com a zona rural, e se estendeu as seguintes sedes municipais: Patos (Latitude $07^{\circ} 01^{\prime} 28^{\prime \prime}$ e Longitude $37^{\circ} 16^{\prime} 48^{\prime \prime}$ ), Cajazeiras (Latitude $-06^{\circ} 53^{\prime} 2^{\prime \prime}$ e Longitude $38^{\circ} 33^{\prime}$ 19"), Sousa (Latitude $-06^{\circ} 45^{\prime} 33^{\prime \prime}$ e Longitude $\left.38^{\circ} 13^{\prime} 41^{\prime \prime}\right)$, Teixeira (Latitude $-07^{\circ} 13^{\prime} 22^{\prime \prime}$ e Longitude $\left.37^{\circ} 15^{\prime} 15^{\prime \prime}\right)$, São Mamede (Latitude $-06^{\circ} 55^{\prime} 36^{\prime \prime}$ e Longitude $37^{\circ} 05^{\prime} 44^{\prime \prime}$ ), São José do Bonfim (Latitude $-07^{\circ} 09^{\prime} 45^{\prime \prime}$ e Longitude $\left.37^{\circ} 18^{\prime} 32^{\prime \prime}\right)$, Santa Luzia (Latitude $-06^{\circ}$ $52^{\prime} 20^{\prime \prime}$ e Longitude $\left.36^{\circ} 55^{\prime} 07^{\prime \prime}\right)$, e Soledade (Lat itude $-07^{\circ} 03^{\prime} 26^{\prime \prime}$ e Longitude $\left.36^{\circ} 21^{\prime} 46^{\prime \prime}\right)$.

Direcionou-se a pesquisa em três vertentes, a saber:

a) Seleção e identificação das espécies de porte arbóreo;

b) Análise das potencialidades paisagísticas;

c) Indicação das espécies para a arborização urbana. 


\section{Seleção e identificação das espécies de porte arbóreo}

No que concerne à origem e características, a vegetação urbana pode ser classificada em três categorias, conforme especificado por Cestaro (1985): Vegetação natural, vegetação introduzida ou implantada, e vegetação espontânea. Dentre estas categorias, direcionou-se prioritariamente os estudos para a vegetação de porte arbóreo com ocorrência natural ou introduzida, porém não descartou a possibilidade de identificação e seleção de algumas espécies inseridas de forma espontânea no ambiente urbano.

As espécies foram selecionadas tomando-se como referência a luminosidade, ou seja, a capacidade que a espécie tem em suportar sol pleno, de acordo com Lorenzi (2002a), Lorenzi (2002b), Gonçalves e Paiva (2004), e Sampaio (2005), em seguida, selecionou-se aquelas que ocorreram em maiores freqüências nas cidades pesquisadas, as quais possuíam as características ideais das árvores urbanas preconizadas por Milano e Dalcin (2000), e Gonçalves e Paiva (2004).

Após essa fase, procedeu-se a pesquisa de campo identificando-se as espécies nas mais variadas paisagens naturais e construídas ocorrentes nas cidades alvo de estudos. Nesta fase, direcionou-se a pesquisa para a identificação das características macromorfológicas das espécies, cujos dados foram registrados por meio de uma planilha padrão (Figura 2) e do registro fotográfico dos espécimes de porte arbóreo, além da coleta de material botânico para identificação e catalogação das espécies desconhecidas. A identificação das espécies desconhecidas foi realizada no Laboratório de Botânica do Campus de Patos da Universidade Federal de Campina Grande, cujas exsicatas encontramse herborizadas e catalogadas no referido laboratório.

Informações complementares sobre o hábito das espécies foram obtidas por informações populares combinada com pesquisas in loco. 
Planilha para coleta de dados.

Fotos: ()Árvore ( ) Tronco ()Ramo ()Flor ()Fruto

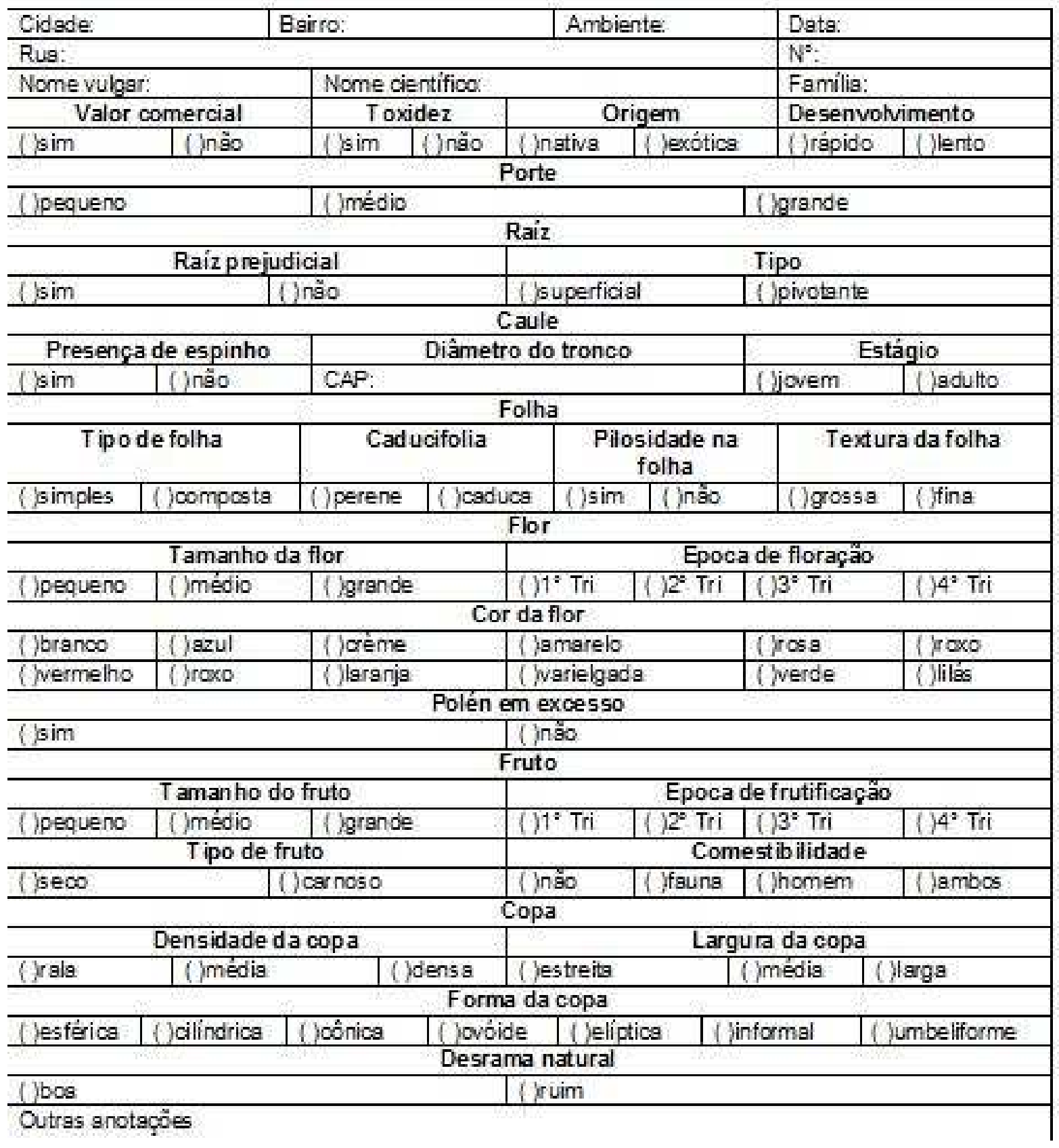

FIGURA 2 - Planilha utilizada para coleta de dados das espécies usadas na arborização urbana em cidades do semi-árido paraibano.

FIGURE 2 - Spreadsheet used for species data collection used in urban forest in cities of Paraibano semi-arid. 


\section{Análise das potencialidades paisagísticas}

Após a identificação das espécies, procedeu-se a análise das suas potencialidades fundamentada em parâmetros estéticos e funcionais.

\section{Características estéticas e funcionais}

As características estéticas e funcionais estudadas conforme Lira Filho (2002), Paiva (2000); Milano e Dalcin (2000) foram cores das flores, época de floração, tipo de fruto, tamanho do fruto, porte das árvores e ritmo de crescimento.

\section{Indicação das espécies para a arborização urbana}

Conforme as características estéticas e funcionais das espécies estudadas, bem como considerando-se o porte das espécies, as mesmas foram indicadas para as seguintes categorias de ambientes urbanos:

a) Arborização de vias públicas;

b) Áreas verdes (parques, praças e jardins);

c) Mata ciliar.

\section{RESULTADOS E DISCUSSÃO}

\section{Identificação das espécies de porte arbóreo}

Foram selecionadas 29 espécies de porte arbóreo, de origem nativa ou exótica adaptada à região, conforme especificado na Tabela 1.

Em relação à vegetação nativa, foram identificadas 13 espécies, enquanto que na de origem exótica computou-se 16 espécies, as quais se inserem nas categorias das arbóreas e palmeiras. Estas espécies estão distribuídas em 17 famílias, sendo as mais freqüentes Mimosaceae (5), Caesalpiniaceae (4), Palmaceae (3) e Bignoniaceae (3).

A falta de planejamento da arborização urbana tem sido uma práxis nas cidades brasileiras, incidindo em erros na escolha das espécies. Medeiros e Lira Filho (2007) elucidam que nessas cidades a maioria das espécies implantadas é de origem exótica. No

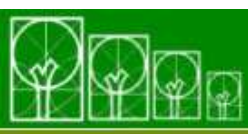


semi-árido brasileiro isto foi constatado por Silva et. al. (2007) que, ao realizarem o inventário da arborização no bairro Centro de Juazeiro-BA, detectaram um percentual muito elevado de uma única espécie - o Ficus benjamina - correspondente a 53,5\%. Por outro lado, estudos realizados por Silveira et. al. (2007) na cidade do Crato-CE, também detectaram um índice elevado de espécies consideradas inadequadas para a arborização urbana, sendo as espécies que apresentaram um maior número de indivíduos foram: Cássia (Senna spectabilis), Mangueira (Mangifera indica), Bejamim-Ficus (Ficus retusa), Três marias (Bougainvillea glabra Choisy), Papoula (Hibiscus rosa sinensis), Jambo (Eugenia malaccensis).

TABELA 1 - Espécies de porte arbóreo identificadas em cidades no semi-árido paraibano.

TABLE 1 - Tree species identified in cities of Paraibano semi-arid.

\begin{tabular}{|c|c|c|c|c|}
\hline № & Nome popular & Nome científico & Família & Origem \\
\hline 01 & Algodão da praia & Hibiscus hiliaceus & Malvaceae & Exótica \\
\hline 02 & Aroeira & Myracrodruon urundeuva & Anacardiaceae & Nativa \\
\hline 03 & Babaçu & Orbignya speciosa & Palmaceae & Nativa \\
\hline 04 & Brasileirinho & Erythrina indica picta & Fabaceae & Exótica \\
\hline 05 & Carnaúba & Copernicia prunifera & Palmaceae & Nativa \\
\hline 06 & Carolina & Adenanthera pavoniana & Mimosaceae & Exótica \\
\hline 07 & Cássia seamea & Senna siamea & Caesalpiniaceae & Exótica \\
\hline 08 & Castanhola & Terminalia catapa & Combretaceae & Exótica \\
\hline 09 & Casuarina & Casuarina equisetifolia & Casuarinaceae & Exótica \\
\hline 10 & Chuva de ouro & Cassia fistula & Caesalpiniaceae & Exótica \\
\hline 11 & Craibeira & Tabebuia aurea & Bignoniaceae & Nativa \\
\hline 12 & Espatódea & Spathodea campanulata & Bignoniaceae & Exótica \\
\hline 13 & Esponjinha & Albizia lebbeck & Mimosaceae & Exótica \\
\hline 14 & Eucalipto & Corymbia citriodora & Myrtaceae & Exótica \\
\hline 15 & Felício & Felícium decipiens & Sapindaceae & Exótica \\
\hline 16 & Flamboyant mirim & Caesalpinia pulcherrima & Mimosaceae & Exótica \\
\hline 17 & Ipê mirim & Tecoma stans & Bignoniaceae & Exótica \\
\hline 18 & Jerivá & Syagrus romanzoffiana & Palmaceae & Nativa \\
\hline 19 & Juazeiro & Ziziphus joazeiro & Rhamnaceae & Nativa \\
\hline 20 & Jucá ou Pau ferro & Caesalpinia ferrea & Caesalpiniaceae & Nativa \\
\hline 21 & Madeira nova & Pterogyne nitens & Mimosaceae & Nativa \\
\hline 22 & Murta & Murraya paniculata & Rutaceae & Exótica \\
\hline 23 & Ním & Azadirachta indica & Meliaceae & Exótica \\
\hline 24 & Pata de vaca & Bauhinia forficata & Caesalpiniaceae & Nativa \\
\hline 25 & Pereiro & Aspidosperma pyrifolium & Apocynaceae & Nativa \\
\hline 26 & Sombreiro & Clitoria racemosa & Fabaceae & Nativa \\
\hline 27 & Tamarindo & Tamarindus indica & Mimosaceae & Exótica \\
\hline 28 & Trapiá & Crateva tapia & Capparaceae & Nativa \\
\hline 29 & Urucum & Bixa orellana & Bixaceae & Nativa \\
\hline
\end{tabular}

Das espécies elencadas na Tabela 1, Medeiros e Lira Filho (2007) também indicaram 
sete delas a serem utilizadas na arborização em cidades do semi-árido do Nordeste brasileiro: Hibiscus tiliaceus, Senna siamea, Terminalia catappa Cássia fistula, Albizia lebbec, Caesalpinia pulcherrima, e Azadirachta indica.

Ressalta-se que, apesar do elevado índice de espécies arbóreas utilizadas na arborização em cidades brasileiras, a escolha planejada, ou não, de algumas nativas constitui-se em avanços na arborização urbana.

\section{Análise das potencialidades paisagísticas}

Um aspecto importante a ser observado quando da escolha de espécies para arborização de vias públicas é o porte das árvores quando adulta. Em relação a este parâmetro, os estudos revelaram que de um total de 29 espécies selecionadas, 8 são de grande porte, 14 de porte médio e 7 de pequeno porte (Tabela 2). Esta é uma classificação um tanto quanto difícil de proceder já que os vegetais vão de porte arbustivo a arbóreo sem se ter como separá-los em medidas, embora, para efeitos práticos alguns consideram árvores de pequeno porte aquelas que atingem um máximo de 9 metros; as de porte médio as que alcançam entre 9 e 20 metros e as de grande porte as que ultrapassam os 20 metros de altura quando adultas (GONÇALVES e PAIVA, 2004). De acordo com Paiva (2000), em canteiros centrais de avenidas e ruas e calçadas largas, pode-se optar por pelo uso de árvores de porte grande ou médio. No entanto, em calçadas estreitas, deve-se optar por espécies de pequeno porte ou médio porte.

TABELA 2 - Características das espécies arbóreas identificadas no semi-árido paraibano, conforme o porte, a cor e o tamanho da inflorescência, para uso na arborização urbana.

TABLE 2 - Characteristics of identified trees in Paraibano semi-arid according to size, color and size of inflorescence, for use in urban forest.

\begin{tabular}{|l|l|l|c|l|c|}
\hline No & Nome popular & Nome científico & \multirow{2}{*}{ Porte } & \multicolumn{2}{|c|}{ Florescimento } \\
\cline { 5 - 6 } & & & \multicolumn{2}{|c|}{ Cor } & Tamanho \\
\hline $\mathbf{0 1}$ & Algodão da praia & Hibiscus hiliaceus & $\mathrm{M}$ & amarela & $\mathrm{M}$ \\
\hline $\mathbf{0 2}$ & Aroeira & Myracrodruon urundeuva & $\mathrm{M}$ & verde claro/amarelas & $\mathrm{P}$ \\
\hline $\mathbf{0 3}$ & Babaçu & Orbignya speciosa & $\mathrm{M}$ & amarela & $\mathrm{P}$ \\
\hline $\mathbf{0 4}$ & Brasileirinho & Erythrina indica picta & $\mathrm{P}$ & vermelha & $\mathrm{M}$ \\
\hline $\mathbf{0 5}$ & Carnaúba & Copernicia prunifera & $\mathrm{M}$ & branca/amarelada & $\mathrm{P}$ \\
\hline $\mathbf{0 7}$ & Carolina & Adenanthera pavoniana & $\mathrm{M}$ & branca/amarelada & $\mathrm{P}$ \\
\hline $\mathbf{0 8}$ & Castanhola & Senna siamea & $\mathrm{M}$ & amarela & $\mathrm{P}$ \\
\hline $\mathbf{0 9}$ & Casuarina & Cerminalia catapa & $\mathrm{G}$ & creme & $\mathrm{P}$ \\
\hline
\end{tabular}




\begin{tabular}{|l|l|l|c|l|c|}
$\mathbf{1 0}$ & Chuva de ouro & Cassia fistula & $\mathrm{M}$ & amarela & $\mathrm{G}$ \\
\hline $\mathbf{1 1}$ & Craibeira & Tabebuia aurea & $\mathrm{G}$ & amarela & $\mathrm{M}$ \\
\hline $\mathbf{1 2}$ & Espatódia & Spathodea campanulata & $\mathrm{G}$ & vermelho alaranjada & $\mathrm{G}$ \\
\hline $\mathbf{1 3}$ & Esponjinha & Albizia lebbeck & $\mathrm{M}$ & verde amarelada & $\mathrm{M}$ \\
\hline $\mathbf{1 4}$ & Eucalipto & Corymbia citriodora & $\mathrm{G}$ & creme & $\mathrm{P}$ \\
\hline $\mathbf{1 5}$ & Felício & Felícium decipiens & $\mathrm{M}$ & alvo esverdeadas & $\mathrm{P}$ \\
\hline $\mathbf{1 6}$ & Flamboyant mirim & Caesalpinia pulcherrima & $\mathrm{P}$ & laranja avermelhada & $\mathrm{M}$ \\
\hline $\mathbf{1 7}$ & Ipê mirim & Tecoma stans & $\mathrm{P}$ & amarela & $\mathrm{M}$ \\
\hline $\mathbf{1 8}$ & Jerivá & Syagrus romanzoffiana & $\mathrm{M}$ & branca/amarelada & $\mathrm{P}$ \\
\hline $\mathbf{1 9}$ & Juazeiro & Ziziphus joazeiro & $\mathrm{G}$ & amarela/esverdeada & $\mathrm{P}$ \\
\hline $\mathbf{2 0}$ & Jucá ou Pau ferro & Caesalpinia ferrea & $\mathrm{M}$ & amarelada & $\mathrm{P}$ \\
\hline $\mathbf{2 1}$ & Madeira nova & Pterogyne nitens & $\mathrm{M}$ & amarelo-pálido & $\mathrm{P}$ \\
\hline $\mathbf{2 2}$ & Murta & Murraya paniculata & $\mathrm{P}$ & branca & $\mathrm{P}$ \\
\hline $\mathbf{2 3}$ & Ním & Azadirachta indica & $\mathrm{M}$ & creme & $\mathrm{P}$ \\
\hline $\mathbf{2 4}$ & Pata de vaca & Bauhinia forficata & $\mathrm{P}$ & branca & $\mathrm{M}$ \\
\hline $\mathbf{2 5}$ & Pereiro & Aspidosperma pyrifolium & $\mathrm{P}$ & alva & $\mathrm{P}$ \\
\hline $\mathbf{2 6}$ & Sombreiro & Clitoria racemosa & $\mathrm{G}$ & lilás & $\mathrm{P}$ \\
\hline $\mathbf{2 7}$ & Tamarindo & Tamarindus indica & $\mathrm{M}$ & amarela & $\mathrm{P}$ \\
\hline $\mathbf{2 8}$ & Trapiá & Crateva tapia & $\mathrm{G}$ & alva & $\mathrm{P}$ \\
\hline $\mathbf{2 9}$ & Urucum & Bixa orellana & $\mathrm{P}$ & lilás & $\mathrm{P}$ \\
\hline
\end{tabular}

Porte da árvore: $\mathrm{P}$ (pequeno), $\mathrm{M}$ (médio), $\mathrm{G}$ (grande). Tamanho da flor: $\mathrm{P}$ (pequeno, $\mathrm{M}$ (médio), G (grande).

Ainda em relação ao porte das árvores, a escolha inadequada das espécies gera situações conflitantes com a fiação nas redes de distribuição de eletricidade. Para Vasconcelos (2000), tais conflitos são freqüentes devendo as concessionárias de distribuição de energia elétrica criarem estratégias para contornar ou conviver com toda essa problemática. Para isto, o referido autor sugere o desenvolvimento de tecnologia e procedimentos para intervir nos componentes do sistema: as árvores e as redes. Uma das alternativas seria o uso de condutores protegidos na rede de média tensão e condutores isolados na de baixa tensão. Essa tecnologia permite o contato temporário entre a árvore e a rede, porém não elimina a necessidade de podas.

A estética das árvores é um fator extremamente importante para a arborização urbana. Deve ser evitado, segundo Paiva (2000), o uso de espécies que produzem flores muito grandes, pois quando caem tornam-se escorregadias e representam perigo para os transeuntes, além da sujeira que acumulam nas ruas. Neste contexto, as espécies indicadas nesta pesquisa apresentam, na sua maioria, flores de tamanho pequeno (20 espécies), conforme especificado na Tabela 2. As de tamanho médio perfazem um total de 7 espécies e apenas 2 são de tamanho grande, que foram recomendadas para plantio em áreas verdes ou como mata ciliar.

No que concerne a época de floração, na Tabela 3 expõe-se um leque de opções

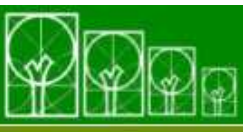

$\mathbf{S} \cdot \mathbf{B} \cdot \mathbf{A} \cdot \mathbf{U}$ Soc. Bras. de Arborização Urbana 
para escolha de espécies, permitindo a obtenção de flores o ano todo, desde que se proceda a uma arborização bem planejada dentro dos padrões técnicos recomendados. Assim, conforme enunciado por Paiva (2000), o tipo de floração e, principalmente, as cores deverão estar de acordo com o entorno, formando um todo agradável na paisagem, em combinação com os elementos construídos e com o restante da vegetação. A coloração das flores varia de tons mais claros até os fortes tais como o vermelho vibrante, as quais podem ser combinadas no plantio das espécies e se obter flores o ano inteiro (Tabela 2).

TABELA 3 - Características das espécies arbóreas identificadas em cidades do semi-árido paraibano, conforme época de floração para uso na arborização urbana.

TABLE 3 - Characteristics of identified tree species in cities of Paraibano semi-arid according to flowering time for use in urban forest.

\begin{tabular}{|c|c|c|c|c|c|c|}
\hline \multirow[t]{2}{*}{ № } & \multirow[t]{2}{*}{ Nome popular } & \multirow[t]{2}{*}{ Nome científico } & \multicolumn{4}{|c|}{ Época de Floração } \\
\hline & & & $1^{\circ}$ Tri & $2^{\circ} \mathrm{Tri}$ & $3^{\circ}$ Tri & $4^{\circ} \mathrm{Tri}$ \\
\hline 01 & Algodão da praia & Hibiscus hiliaceus & $x$ & $x$ & $x$ & $x$ \\
\hline 02 & Aroeira & Myracrodruon urundeuva & & & $x$ & \\
\hline 03 & Babaçu & Orbignya speciosa & $\mathrm{x}$ & & & $\mathrm{x}$ \\
\hline 04 & Brasileirinho & Erythrina indica picta & & $\mathrm{x}$ & $\mathrm{x}$ & \\
\hline 05 & Carnaúba & Copernicia prunifera & & & $\mathrm{x}$ & $\mathrm{x}$ \\
\hline 06 & Carolina & Adenanthera pavoniana & & $\mathrm{x}$ & & \\
\hline 07 & Cássia seamea & Senna siamea & $\mathrm{x}$ & $\mathrm{x}$ & & \\
\hline 08 & Castanhola & Terminalia catapa & $\mathrm{x}$ & & & $\mathrm{x}$ \\
\hline 09 & Casuarina & Casuarina equisetifolia & - & - & - & - \\
\hline 10 & Chuva de ouro & Cassia fistula & $\mathrm{x}$ & & & $\mathrm{x}$ \\
\hline 11 & Craibeira & Tabebuia aurea & & & & $\mathrm{x}$ \\
\hline 12 & Espatódia & Spathodea campanulata & $\mathrm{x}$ & $\mathrm{x}$ & $\mathrm{x}$ & $\mathrm{x}$ \\
\hline 13 & Esponjinha & Albizia lebbeck & & & $\mathrm{x}$ & $\mathrm{x}$ \\
\hline 14 & Eucalipto & Corymbia citriodora & & $\mathrm{x}$ & & \\
\hline 15 & Felício & Felícium decipiens & & & $\mathrm{x}$ & \\
\hline 16 & Flamboyant mirim & Caesalpinia pulcherrima & $\mathrm{x}$ & & & $\mathrm{x}$ \\
\hline 17 & Ipê mirim & Tecoma stans & $\mathrm{x}$ & & & $\mathrm{x}$ \\
\hline 18 & Jerivá & Syagrus romanzoffiana & $x$ & & & $x$ \\
\hline 19 & Juazeiro & Ziziphus joazeiro & $x$ & & & $x$ \\
\hline 20 & Jucá ou Pau ferro & Caesalpinia ferrea & $x$ & & & $x$ \\
\hline 21 & Madeira nova & Pterogyne nitens & $x$ & $x$ & & \\
\hline 22 & Murta & Murraya paniculata & & $x$ & $x$ & \\
\hline 23 & Ním & Azadirachta indica & & & $x$ & \\
\hline 24 & Pata de vaca & Bauhinia forficata & & & $x$ & $x$ \\
\hline 25 & Pereiro & Aspidosperma pyrifolium & $x$ & $x$ & & \\
\hline 26 & Sombreiro & Clitoria racemosa & & & & $\mathrm{x}$ \\
\hline 27 & Tamarindo & Tamarindus indica & & & & $x$ \\
\hline 28 & Trapiá & Crateva tapia & & & & $x$ \\
\hline 29 & Urucum & Bixa orellana & $x$ & & & $x$ \\
\hline
\end{tabular}

Tri: Trimestre 
Quanto aos frutos, 93\% das espécies indicadas na Tabela 4 encontram-se nos padrões desejáveis para plantio em ruas e avenidas, cujo tamanho varia de pequeno a médio. Para ruas e canteiros centrais não é recomendado o uso de espécies com frutos grandes, pois estes podem representar perigo para os pedestres e para os veículos estacionados nas vias públicas (MILANO e DALCIN, 2000; GONÇALVES e PAIVA, 2004).

TABELA 4 - Características das espécies arbóreas identificadas em cidades do semi-árido paraibano, conforme ritmo de crescimento e frutificação.

TABLE 4 - Characteristics of identified tree species in cities of Paraibano semi-arid according to rate of growth and fruiting.

\begin{tabular}{|c|c|c|c|c|c|}
\hline \multirow[t]{2}{*}{ № } & \multirow{2}{*}{ Nome popular } & \multirow{2}{*}{ Nome científico } & \multirow{2}{*}{ Crescimento } & \multicolumn{2}{|c|}{ Frutescência } \\
\hline & & & & Tipo & Tamanho \\
\hline 01 & Algodão da praia & Hibiscus hiliaceus & rápido & seco & $P$ \\
\hline 02 & Aroeira & Myracrodruon urundeuva & moderado & carnoso & $P$ \\
\hline 03 & Babaçu & Orbignya speciosa & lento & carnoso & $M$ \\
\hline 04 & Brasileirinho & Erythrina indica picta & rápido & seco & $M$ \\
\hline 05 & Carnaúba & Copernicia prunifera & lento & carnoso & $\mathrm{P}$ \\
\hline 06 & Carolina & Adenanthera pavoniana & moderado & seco & $M$ \\
\hline 07 & Cássia seamea & Senna siamea & rápido & seco & M \\
\hline 08 & Castanhola & Terminalia catapa & rápido & carnoso & $\mathrm{M}$ \\
\hline 09 & Casuarina & Casuarina equisetifolia & lento & seco & $\mathrm{M}$ \\
\hline 10 & Chuva de ouro & Cassia fistula & rápido & seco & G \\
\hline 11 & Craibeira & Tabebuia aurea & lento & seco & $\mathrm{M}$ \\
\hline 12 & Espatódia & Spathodea campanulata & rápido & seco & G \\
\hline 13 & Esponjinha & Albizia lebbeck & rápido & seco & $\mathrm{M}$ \\
\hline 14 & Eucalipto & Corymbia citriodora & rápido & seco & $\mathrm{P}$ \\
\hline 15 & Felício & Felícium decipiens & moderado & seco & M \\
\hline 16 & Flamboyant mirim & Caesalpinia pulcherrima & rápido & seco & $P$ \\
\hline 17 & Ipê mirim & Tecoma stans & rápido & seco & M \\
\hline 18 & Jerivá & Syagrus romanzoffiana & lento & carnoso & $\mathrm{P}$ \\
\hline 19 & Juazeiro & Ziziphus joazeiro & lento & carnoso & $P$ \\
\hline 20 & Jucá ou Pau ferro & Caesalpinia ferrea & moderado & seco & $P$ \\
\hline 21 & Madeira nova & Pterogyne nitens & rápido & seco & $P$ \\
\hline 22 & Murta & Murraya paniculata & lento & seco & $P$ \\
\hline 23 & Ním & Azadirachta indica & rápido & carnoso & $P$ \\
\hline 24 & Pata de vaca & Bauhinia forficata & rápido & seco & M \\
\hline 25 & Pereiro & Aspidosperma pyrifolium & lento & seco & $P$ \\
\hline 26 & Sombreiro & Clitoria racemosa & rápido & seco & M \\
\hline 27 & Tamarindo & Tamarindus indica & moderado & carnoso & M \\
\hline 28 & Trapiá & Crateva tapia & lento & carnoso & $\mathrm{M}$ \\
\hline 29 & Urucum & Bixa orellana & rápido & seco & $\mathrm{M}$ \\
\hline
\end{tabular}

Tamanho do Fruto: P (pequeno), M (médio), G (grande).

Dentre as espécies pesquisadas, alguns frutos são comestíveis pelo ser humano e também pela fauna, a exemplo da Terminalia catapa, Ziziphus joazeiro, Tamarindus indica, 
entre outras. Outras partes dessas espécies também são utilizadas para diversos fins como, por exemplo, as sementes da Adenanthera pavoniana no artesanato, as folhas da Copernicia prunifera para obtenção da "cera de carnaúba", lubrificante para sapatos, vernizes, sabonetes, etc. A Tabebuia aurea é muito útil para reflorestamentos mistos de áreas degradadas destinadas à recomposição da vegetação. A Senna siamea é muito encontrada na arborização de ruas, enquanto a Bixa orellana é muito utilizada como repelente e sua madeira é usada para lenha, além das suas sementes que são condimentares e tintoriais. Já a Azadirachta indica é uma espécie que possui um bom desenvolvimento em altas temperaturas, tendo se adaptado perfeitamente na região e recentemente muito utilizada na arborização.

$\mathrm{Na}$ indicação das espécies selecionadas também se levou em consideração o ritmo de crescimento conforme especificado a Tabela 4. Pode-se verificar que $70 \%$ delas enquadram-se na categoria de crescimento rápido e moderado que, segundo Gonçalves e Paiva (2004) são desejáveis para plantio em ruas e avenidas.

\section{Indicação das espécies para a arborização urbana}

Do total de espécies identificadas, vinte e uma foram indicadas para arborização de vias públicas, vinte e nove para áreas verdes e sete para mata ciliar, conforme especificado na Tabela 5.

TABELA 5 - Características das espécies arbóreas identificadas em cidades do semi-árido paraibano, conforme sua indicação na arborização urbana.

TABLE 5 - Characteristics of identified tree species in cities of Paraibano semi-arid according to its indication for urban forest.

\begin{tabular}{|l|l|l|l|}
\hline № & Nome popular & Nome científico & Indicação \\
\hline 01 & Algodão da praia & Hibiscus hiliaceus & AR, AV \\
\hline 02 & Aroeira & Myracrodruon urundeuva & AR, AV \\
\hline 03 & Babaçu & Orbignya speciosa & AR, AV \\
\hline 04 & Brasileirinho & Erythrina indica picta & AR, AV \\
\hline 05 & Carnaúba & Copernicia prunifera & AR, AV \\
\hline 06 & Carolina & Adenanthera pavoniana & AR, AV \\
\hline 07 & Cássia seamea & Senna siamea & AR, AV \\
\hline 08 & Castanhola & Terminalia catapa & AV \\
\hline 09 & Casuarina & Casuarina equisetifolia & AV, MC \\
\hline 10 & Chuva de ouro & Cassia fistula & AV \\
\hline 11 & Craibeira & Tabebuia aurea & AV, MC \\
\hline 12 & Espatódia & Spathodea campanulata & AV \\
\hline
\end{tabular}




\begin{tabular}{|c|c|c|c|}
\hline 13 & Esponjinha & Albizia lebbeck & $\mathrm{AR}, \mathrm{AV}$ \\
\hline 14 & Eucalipto & Corymbia citriodora & AV \\
\hline 15 & Felício & Felícium decipiens & $\mathrm{AR}, \mathrm{AV}$ \\
\hline 16 & Flamboyant mirim & Caesalpinia pulcherrima & $\mathrm{AR}, \mathrm{AV}$ \\
\hline 17 & Ipê mirim & Tecoma stans & $\mathrm{AR}, \mathrm{AV}$ \\
\hline 18 & Jerivá & Syagrus romanzoffiana & $\mathrm{AR}, \mathrm{AV}$ \\
\hline 19 & Juazeiro & Ziziphus joazeiro & $\mathrm{AR}, \mathrm{AV}, \mathrm{MC}$ \\
\hline 20 & Jucá ou Pau ferro & Caesalpinia ferrea & $\mathrm{AR}, \mathrm{AV}$ \\
\hline 21 & Madeira nova & Pterogyne nitens & $\mathrm{AR}, \mathrm{AV}$ \\
\hline 22 & Murta & Murraya paniculata & $\mathrm{AR}, \mathrm{AV}$ \\
\hline 23 & Ním & Azadirachta indica & $\mathrm{AR}, \mathrm{AV}$ \\
\hline 24 & Pata de vaca & Bauhinia forficata & $\mathrm{AR}, \mathrm{AV}, \mathrm{MC}$ \\
\hline 25 & Pereiro & Aspidosperma pyrifolium & $\mathrm{AR}, \mathrm{AV}, \mathrm{MC}$ \\
\hline 26 & Sombreiro & Clitoria racemosa & AV \\
\hline 27 & Tamarindo & Tamarindus indica & $\mathrm{AR}, \mathrm{AV}, \mathrm{MC}$ \\
\hline 28 & Trapiá & Crateva tapia & $\mathrm{AV}, \mathrm{MC}$ \\
\hline 29 & Urucum & Bixa orellana & $\mathrm{AR}, \mathrm{AV}$ \\
\hline
\end{tabular}

Indicação: AR (arborização de ruas), AV (áreas verdes), MC (Mata ciliar).

Das espécies pesquisadas cinco são indicadas apenas para áreas verdes (Terminalia catapa, Cassia fistula, Spathodea campanulata, Corymbia citriodora e Clitoria racemosa), três para áreas verdes e mata ciliar (Casuarina equisetifolia, Tabebuia aurea e Crateva tapia), e quatro delas podem ser plantadas em áreas verdes, nas ruas e também como mata ciliar (Ziziphus joazeiro, Bauhinia forficata, Aspidosperma pyrifolium e Tamarindus indica). As demais espécies podem ser plantadas tanto em áreas verdes quanto na arborização de vias públicas.

Algumas espécies selecionadas já foram indicadas para a arborização urbana em trabalhos anteriores. Pesquisas realizadas no semi-árido por Lira Filho et al. (1994), desenvolvidas em áreas periféricas da cidade de Patos/PB, também indicaram as espécies Senna siamea e Albizia lebbeck para a arborização das vias públicas. Por outro lado, Albuquerque (1985), em trabalho semelhante a este, também indicou as espécies Senna siamea, Tabebuia áurea, Ziziphus joazeiro e Tamarindus indica, para arborização de Patos/PB.

$\mathrm{Na}$ arborização de vias publicas, as palmeiras são recomendadas para canteiro central em avenidas (CEMIG, 2001), porém também podem ser utilizadas em projetos paisagísticos de áreas verdes urbana. Para Eymael e Barreto (2007) as palmeiras constituem elementos determinantes para a criação de ambientes tropicais, sendo utilizadas em parques, praças, jardins públicos ou privados e ocupam uma posição relevante na paisagem do Recife, uma vez que a partir de um levantamento em 16 praças desta cidade, foram selecionadas 12 espécies de origem nativa e exóticas. No que concerne as espécies 
indicadas para o semi-árido, das três espécies citadas na Tabela 5, encontram-se duas delas (Orbignya speciosa e Syagrus romanzoffiana) nos estudos realizados por Eymael e Barreto (2007). Com isto, constata-se que as referidas palmeiras se adéquam a arborização urbana do Nordeste, adaptando-se aos climas quentes, sejam eles seco ou úmido.

\section{CONCLUSÕES}

Sob condições de semi aridez existe um grande número de espécies que podem ser utilizadas na arborização urbana. As espécies identificadas apresentaram uma variabilidade em relção ao seu porte, podendo ser recomendadas tanto para áreas verdes ou como mata ciliar.

Com isto detectou-se e a importância das árvores urbanas para o conforto ambiental nas cidades do semi-árido brasileiro, as quais contribuirão para elevação do padrão sócioambiental, cujos resultados trarão benefícios ecológicos, econômicos e sociais.

\section{REFERÊNCIAS BIBLIOGRÁFICAS}

ALBUQUERQUE, S. F. Indicação das espécies para arborização de ruas na cidade de Patos-Paraíba. 1985. 47f. Monografia (Graduação em Engenharia Florestal) - Centro de Saúde e Tecnologia Rural, Universidade Federal de Campina Grande, Patos, 1985.

CESTARO, L. A. A Vegetação no ecossistema urbano. In: ENCONTRO NACIONAL SOBRE ARBORIZAÇÃO URBANA, 1, 1985. Anais... Porto Alegre: SBAU, 1985. p. 51-56.

COMPANHIA ENERGÉTICA DE MINAS GERAIS/ CEMIG. Manual de arborização. Belo Horizonte: CEMIG, 2001. 40 p.

CODETER- Coordenação de Desenvolvimento Territorial do Médio Sertão. Plano territorial de desenvolvimento sustentável. Patos: MDA/SDT, 2005. $104 \mathrm{p}$.

EYMAEL, P. P.; BARRETO, R. C. Palmeiras mais representativas na paisagem da cidade do Recife. In: REUNIÃO NORDESTINA DE BORTÂNICA, 30․ Crato de 04 a 07 de julho de 2007. Anais... Crato-CE: SBB, URCA, 2007. p. 14

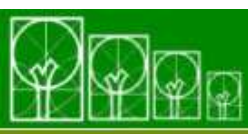

S $\cdot \mathbf{B} \cdot \mathbf{A} \cdot \mathbf{U}$ Soc. Bras. de Arborização Urbana 
GONÇALVES, W.; PAIVA, H.N., Árvores para o ambiente urbano. Viçosa: Aprenda Fácil, 2004. 243 p. (Coleção Jardinagem e Paisagismo. Série Arborização Urbana, 3).

LIRA FILHO, J. A.; ALENCAR, G. V.; MOREIRA, J. M. Arborização urbana periférica em Patos- PB. In: XVIII REUNIÃO NORDESTINA BOTÂNICA, 47., 1994, Paraíba. Anais... Paraíba: Simuramba Versicolor, 1994. p. 206.

LIRA FILHO, J. A., Paisagismo: elementos de composição e estética. Viçosa: Aprenda Fácil, 2002. 194 p. 9 (Coleção Jardinagem e Paisagismo. Série Planejamento Paisagísticos, 2).

LORENZI, H. Árvores brasileiras: manual de identificação e cultivo de plantas arbóreas. 4 ed. Nova Odessa, SP: Instituto Plantarum, v.1, 2002. 368 p.

Árvores brasileiras: manual de identificação e cultivo de plantas arbóreas. 4 ed. Nova Odessa,SP: Instituto Plantarum, v.4, 2002. 368 p.

MEDEIROS, M. A. S.; LIRA FILHO, J. A. Indicação de espécies arbóreas adaptadas ao semi-árido brasileiro, para o paisagismo urbano. In: REUNIÃO NORDESTINA DE BORTÂNICA, 30․ Crato de 04 a 07 de julho de 2007. Anais... Crato, CE: SBB, URCA, 2007. p. 26

MILANO, M.; DALCIN, E., Arborização de vias públicas. Rio de Janeiro: Ligth, 2000. 206 p.

PAIVA, H.N. Seleção de espécies para arborização urbana. Revista Ação Ambiental, v.2, n. 9, p. 14-16, 2000.

SILVA, R. M. et al. Caracterização da arborização do bairro centro da cidade de Juazeiro. In: REUNIÃO NORDESTINA DE BORTÂNICA, 30․ Crato de 04 a 07 de julho de 2007. Anais... Crato-CE: SBB, URCA, 2007. p. 44

SILVEIRA, A. L. et al. Plantas ornamentais utilizadas na arborização do município de CratoCE. In: REUNIÃO NORDESTINA DE BORTÂNICA, 30․ Crato de 04 a 07 de julho de 2007. Anais... Crato-CE: SBB, URCA, 2007. p. 47 
SAMPAIO, E.V.S.B. Espécies da flora nordestina de importância econômica potencial. Recife: APN, 2005. 331 p.

SUDEMA - Superintendência de Administração do Meio Ambiente. Atualização do diagnóstico florestal do Estado da Paraíba. João Pessoal: SUDEMA, 2004. 268p.

VASCONCELOS, A. Entrevista da CEMIG. Revista Ação Ambiental, v.2, n. 9, p. 5-6, 2000. 